\title{
Effect of spin-orbit couplings in graphene with and without potential modulation
}

\author{
Kh. Shakouri, ${ }^{1,}$ M. Ramezani Masir, ${ }^{1}$ A. Jellal, ${ }^{2,3,4, \dagger}$ E. B. Choubabi, ${ }^{4}$ and F. M. Peeters ${ }^{1}$ \\ ${ }^{1}$ Departement Fysica, Universiteit Antwerpen Groenenborgerlaan 171, B-2020 Antwerpen, Belgium \\ ${ }^{2}$ Saudi Center for Theoretical Physics, Dhahran, Saudi Arabia \\ ${ }^{3}$ Physics Department, College of Sciences, King Faisal University, P.O. Box 380, Alahssa 31982, Saudi Arabia \\ ${ }^{4}$ Theoretical Physics Group, Faculty of Sciences, Chouaïb Doukkali University, 24000 El Jadida, Morocco
}

(Received 28 June 2013; revised manuscript received 30 July 2013; published 4 September 2013)

\begin{abstract}
We investigate the effect of Rashba and intrinsic spin-orbit couplings on the electronic properties and spin configurations of Dirac fermions confined in: (i) a flat graphene sheet, (ii) a graphene wire with $p-n-p$ structure, and (iii) a superlattice of graphene wires. The interplay between the spin-orbit interaction mechanisms breaks the electron-hole symmetry and the spin configuration induced by Rashba spin-orbit coupling lacks inversion symmetry in $k$ space. We show that the Rashba spin-orbit interaction doubles the Fabry-Pérot resonant modes in the transmission spectrum of a graphene wire and opens new channels for the electron transmission. Moreover, it leads to the appearance of spin split extra Dirac cones in the energy spectrum of a graphene superlattice. It is shown that the spin of the electrons and holes confined in a flat graphene sheet is always perpendicular to their motion while this is not the case for the other nanostructures.
\end{abstract}

DOI: 10.1103/PhysRevB.88.115408

PACS number(s): 81.05.ue, 71.70.Ej

\section{INTRODUCTION}

Spintronics is an emerging field that has received great attention in recent years. The strong research interest in spintronics is due to its promising applications, in particular to achieve tiny devices that are expected to work more adaptably and faster than today's electronic devices. ${ }^{1}$ The realization of stable single-layer carbon crystals, called graphene, as well as that of multilayer graphene has triggered an avalanche of interest in graphene's properties. ${ }^{2,3}$ Graphene has excellent electrical and thermal properties, e.g., massless and chiral Dirac fermions, which move with a Fermi velocity of about c/300, and a linear spectrum close to the $K$ and $K^{\prime}$ points. ${ }^{2,3}$ Furthermore, the spin relaxation time in graphene is long due to the absence of hyperfine coupling and the very small intra-atomic spin-orbit coupling (SOC) in carbon atoms. This makes single-layer as well as few-layer graphene promising candidates for spin-based devices, where the spin-orbit couplings play a key role in spin relaxation, ${ }^{4-6}$ spin injection, ${ }^{7,8}$ and spin transport. ${ }^{4,9-12}$

In recent years there has been an increasing interest in the study of intrinsic and Rashba spin-orbit interaction mechanisms in graphene and also its derivatives, ${ }^{13-16}$ which are respectively due to the intra-atomic spin-orbit interaction of carbon atoms ${ }^{17-19}$ and the out-of-plane inversion asymmetry of the graphene sheet. ${ }^{18}$ The intrinsic spin-orbit splitting in pristine graphene is very weak, about $0.01-0.05 \mathrm{meV},{ }^{17,20}$ which is too small for practical purposes. However, by taking the intrinsic spin-orbit coupling into account, a masslike term with opposite sign in the two Dirac cones opens a gap in the spectrum. This turns graphene from a semimetal into a quantum spin-Hall insulator with quantized spin-Hall conductance. ${ }^{13}$ Using angle-resolved photoemission spectroscopy it was shown that the Rashba splitting of the $\pi$ bands in graphene when deposited on $\mathrm{Ni}$ (111) is about $225 \mathrm{meV}^{21}$ This result was challenged later by Rader et al. ${ }^{22}$ however, the possibility to achieve a giant Rashba spin-orbit splitting (near $100 \mathrm{meV}$ ) was still supported by first-principles calculations. ${ }^{23}$ It was predicted that doping graphene with $5 \mathrm{~d}$ transition metals induces a large spin-orbit interaction, which results in an anomalous quantum Hall effect. ${ }^{16} \mathrm{~A}$ recent experiment showed that a strong spin-orbit coupling is achievable by the intercalation of $\mathrm{Au}$ atoms at the interface of graphene-Ni in which the hybridization with the Au $5 \mathrm{~d}$ orbitals causes a large spin-orbit splitting up to $100 \mathrm{meV}^{24}$ It is also known that impurities $^{25}$ and lattice distortions ${ }^{26}$ comprise other sources of SOCs.

In this paper, we present an analytical study of the effect of spin-orbit couplings on the electronic and spin-related properties of low-energy fermions confined in: (i) a flat graphene sheet, (ii) a graphene wire, and (iii) a superlattice of graphene wires. The latter two structures can be realized artificially by using external electrical gates. For all these structures the energy dispersion relation of low-energy Dirac fermions is derived. The spin-chiral states induced by Rashba SOC result in a spin configuration that lacks inversion symmetry in $k$ space. We show that the spin of a free electron that travels in a flat graphene sheet (with zero external electrostatic potential) is perpendicular to its motion while the application of an external electrostatic potential causes the spin to have a component parallel to the electron motion. It is also demonstrated that the Rashba SOC induces extra Fabry-Pérot resonant modes, providing additional channels for the electron transmission across a graphene wire. Moreover, it leads to the appearance of spin split extra Dirac cones in the energy spectrum of a superlattice of graphene wires and, as a result, new oscillating structure emerges in the spectrum of the density of states as well as in the diffusive conductivity.

The paper is organized as follows. In Sec. II we describe our model Hamiltonian and investigate the effect of spin-orbit couplings in a flat graphene sheet. In Sec. III we deal with the transmission and the bound states of an electron that is confined in a graphene wire. In Sec. IV we investigate the effect of spin-orbit couplings on a superlattice of graphene wires. Our conclusions are summarized in Sec. V.

\section{SPIN-ORBIT HAMILTONIAN}

The effective two-band Hamiltonian of a pristine graphene layer in the presence of spin-orbit interactions can be divided 
into three terms

$$
H_{\text {eff }}=H_{0}+H_{I}+H_{R} .
$$

Here $H_{0}$ is the usual Dirac Hamiltonian, $H_{I}$ is the intrinsic spin-orbit Hamiltonian with the following standard form

$$
H_{I}=\Delta \tau \sigma_{z} s_{z},
$$

where $\Delta$ determines the strength of the intrinsic SOC, $\sigma$, and $s$ are the pseudospin and real spin Pauli matrices, and $\tau= \pm 1$ denote the $K$ and $K^{\prime}$ valleys. The intrinsic SOC term plays the same role as the mass term in the Dirac Hamiltonian. The third term in (1) comes from an extrinsic spin-orbit interaction ${ }^{19}$

$$
H_{R}=\lambda\left(\tau \sigma_{x} s_{y}-\sigma_{y} s_{x}\right),
$$

$$
\left(\begin{array}{ccc}
\bar{\Delta} & -i \partial_{x}-i k_{y} & 0 \\
-i \partial_{x}+i k_{y} & -\bar{\Delta} & -i \bar{\lambda} \\
0 & i \bar{\lambda} & -\bar{\Delta} \\
0 & 0 & -i \partial_{x}+i k_{y}
\end{array}\right.
$$

where $\bar{\Delta}=\frac{L}{v_{F} \hbar} \Delta, L k_{y} \rightarrow k_{y}, \bar{\lambda}=\frac{L}{v_{F} \hbar} \lambda, \varepsilon=\frac{L}{v_{F} \hbar} E$, and $v=$ $\frac{L}{v_{F} \hbar} V$. We rewrite the Dirac equation as a system of coupled equations

$$
\begin{aligned}
-i L^{+} \psi_{B, \uparrow} & =(\varepsilon-v-\bar{\Delta}) \psi_{A, \uparrow} \\
-i L^{-} \psi_{A, \uparrow} & =(\varepsilon-v+\bar{\Delta}) \psi_{B, \uparrow}+i \bar{\lambda} \psi_{A, \downarrow} \\
-i L^{+} \psi_{B, \downarrow} & =(\varepsilon-v+\bar{\Delta}) \psi_{A, \downarrow}-i \bar{\lambda} \psi_{B, \uparrow} \\
-i L^{-} \psi_{A, \downarrow} & =(\varepsilon-v-\bar{\Delta}) \psi_{B, \downarrow},
\end{aligned}
$$

where $L^{ \pm}=d / d x \pm k_{y}$. In the case of constant potential, i.e., $L^{ \pm} v=0$, we readily obtain

$$
\left[L^{+} L^{-}+(\varepsilon-v)^{2}-\bar{\Delta}^{2}\right] \psi_{A, \uparrow}=-\bar{\lambda} L^{+} \psi_{A, \downarrow},
$$

and

$$
\left[L^{+} L^{-}+(\varepsilon-v)^{2}-\bar{\Delta}^{2}\right] \psi_{A, \downarrow}=i(\varepsilon-v-\bar{\Delta}) \bar{\lambda} \psi_{B, \uparrow} .
$$

By multiplying the operator $L^{+}$on both sides in Eq. (8) and using Eqs. (6) and (7) we find

$$
\left[\frac{d^{2}}{d x^{2}}-k_{y}^{2}+(\varepsilon-v)^{2}-\bar{\Delta}^{2} \pm \bar{\lambda}(\varepsilon-v-\bar{\Delta})\right] \psi_{A, \uparrow}=0 .
$$

After introducing the momentum

$$
k_{ \pm}^{2}=-k_{y}^{2}+(\varepsilon-v)^{2}-\bar{\Delta}^{2} \pm \bar{\lambda}(\varepsilon-v-\bar{\Delta}),
$$

we find the energy dispersion

$$
\varepsilon=v+p \sqrt{k_{ \pm}^{2}+k_{y}^{2}+\bar{\Delta}^{2}+\frac{\bar{\lambda}^{2}}{4} \pm \bar{\lambda} \bar{\Delta}} \mp \frac{\bar{\lambda}}{2},
$$

for both $p=1$ and $p=-1$. In order to find the associated eigenspinors, let us first define the phases $\theta_{ \pm}$as follows

$$
e^{i \theta_{ \pm}}=\frac{k_{y}+i k_{ \pm}}{\left|k_{y}+i k_{ \pm}\right|}=\frac{k_{y}+i k_{ \pm}}{|\varepsilon(\bar{\Delta}=0, \bar{\lambda}=0)-v|} .
$$

which is known as Rashba SOC. This term couples the spin and pseudospin degrees of freedom by introducing off diagonal terms to the Hamiltonian matrix. In Eq. (3), $\lambda$ is the strength of the Rashba spin-orbit interaction. We rewrite the two-dimensional Dirac Hamiltonian around the $\mathbf{K}$ point as

$$
\begin{aligned}
H= & \mathbf{1} \otimes\left[v_{F} \vec{\sigma} \cdot \vec{p}+V(x) \mathbf{1}\right]+\frac{\lambda}{2}\left(s_{y} \otimes \sigma_{x}-s_{x} \otimes \sigma_{y}\right) \\
& +\Delta s_{z} \otimes \sigma_{z},
\end{aligned}
$$

where $v_{F} \approx 10^{6} \mathrm{~m} / \mathrm{s}$ is the Fermi velocity of the electron, and $V(x)$ is the applied electrostatic potential along the $x$ axis.

The Hamiltonian is invariant under an arbitrary translation along the $y$ direction which allows to apply the ansatz $\Psi(x, y)=e^{i k_{y} x} \psi(x)$. We switch to a dimensionless form of the Dirac equation by replacing $x / L \rightarrow x$,

$$
\left.\begin{array}{c}
0 \\
0 \\
-i \partial_{x}-i k_{y} \\
\bar{\Delta}
\end{array}\right)\left(\begin{array}{l}
\psi_{A, \uparrow} \\
\psi_{B, \uparrow} \\
\psi_{A, \downarrow} \\
\psi_{B, \downarrow}
\end{array}\right)=(\varepsilon-v)\left(\begin{array}{l}
\psi_{A, \uparrow} \\
\psi_{B, \uparrow} \\
\psi_{A, \downarrow} \\
\psi_{B, \downarrow}
\end{array}\right),
$$

Now, the propagating plane waves can be plainly written as $\psi\left(k_{ \pm}\right)=A_{ \pm} e^{i k_{ \pm} x}$ where $A_{ \pm}$is a four-component spinor given by

$$
A_{ \pm}=\left(\begin{array}{c}
e^{i \theta_{ \pm}} \\
i c_{ \pm} \\
\pm c_{ \pm} \\
\pm i e^{-i \theta_{ \pm}}
\end{array}\right)
$$

and $c_{ \pm}=(\varepsilon-v-\bar{\Delta}) /\left|k_{ \pm}^{2}+k_{y}^{2}\right|^{1 / 2}$

Electron-hole energy dispersion. Figure 1 shows the lowenergy band dispersion of Dirac fermions traveling freely in the $x$ direction $\left(k_{y}=0\right)$ in the absence of any applied electrostatic potential $(v=0)$. The strengths of the intrinsic and Rashba SOCs are $\bar{\Delta}=0.01$ and $\bar{\lambda}=1.52$, which correspond to the SOC strengths $\Delta \approx 0.05 \mathrm{meV}$ and $\lambda=10 \mathrm{meV}$ with the length scale $L=100 \mathrm{~nm}$. In the case of only pure intrinsic SOC, no spin-orbit splitting occurs for the Dirac cone but a gap opens up between the electron-hole energy bands [Fig. 1(a)]. In contrast, Rashba SOC splits the energy bands and removes the energy gap. Once the strengths of the Rashba and intrinsic SOCs become comparable the symmetry of the electron-hole energy dispersion is broken [see Fig. 1(b)]. This electron-hole symmetry is retained if only one SOC mechanism is present.

In order to attribute a physical meaning to the $k_{ \pm}$wave vectors we examine the spin configuration of the electrons occupying the corresponding spin split band of the $k_{ \pm}$states. We have

$$
\frac{\left\langle\psi\left(k_{ \pm}\right)|\hat{S}| \psi\left(k_{ \pm}\right)\right\rangle}{\left\langle\psi\left(k_{ \pm}\right) \mid \psi\left(k_{ \pm}\right)\right\rangle}=\frac{\hbar c_{ \pm}}{1+c_{ \pm}^{2}}\left(\begin{array}{c} 
\pm \cos \theta_{ \pm} \\
\mp \sin \theta_{ \pm} \\
0
\end{array}\right),
$$

which shows that the real spin of an electron occupying the $k_{+}$state is in the opposite direction to the electron of the $k_{-}$ state. Moreover the spin is in-plane and is perpendicular to the electron momentum, which is the same as to an electron spin 

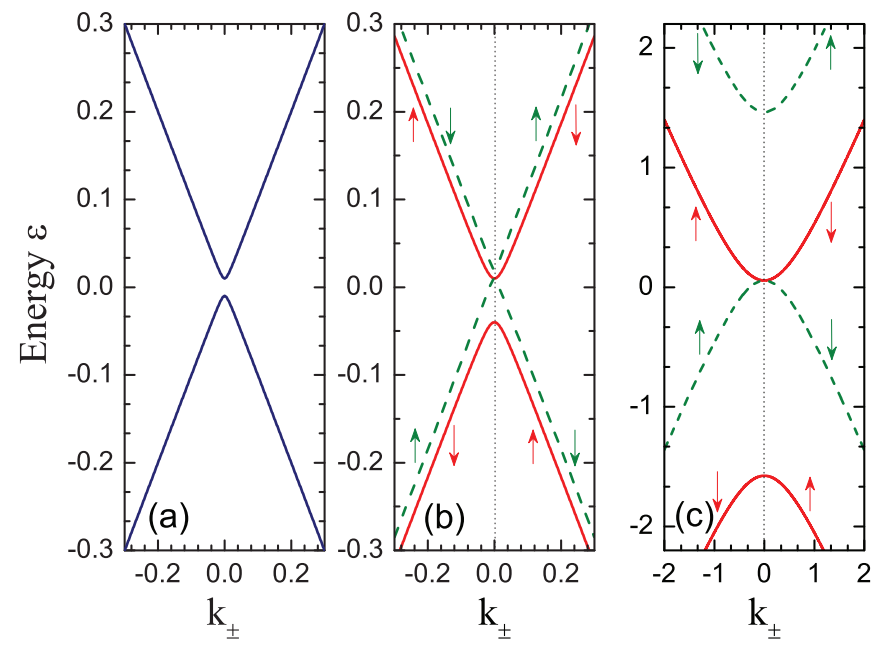

FIG. 1. (Color online) The energy dispersion of free electrons and holes in a flat graphene sheet for $k_{y}=0, v=0$ and (a) $\bar{\Delta}=0.01$, $\bar{\lambda}=0$, (b) $\bar{\Delta}=0.01, \bar{\lambda}=0.03$, and (c) $\bar{\Delta}=0, \bar{\lambda}=1.52$. The solid (dashed) curves correspond to the $k_{+}\left(k_{-}\right)$wave vector, which refer to the different spin-chiral states. The arrows indicate the spin-up and spin-down states. By changing the direction of motion a spin inversion occurs.

confined in a two-dimensional electron gas in the presence of Rashba SOC. Figure 2 shows this result for electrons situating in two different energy bands corresponding to $k_{ \pm}$states. The Rashba SOC induces new spin-chiral states with a spin configuration obeying the relation $\hat{S}(-\vec{k})=-\hat{S}(\vec{k})$, which lacks inversion symmetry. Note that if the electron-hole energy band is symmetric, the electron spin is opposite to the spin of the corresponding hole traveling in opposite direction [see Fig. 1(c)]. This implies that in the presence of Rashba SOC the spin of the holes in $p$-doped graphene is opposite to the spin of electrons with the same energy in $n$-doped graphene.

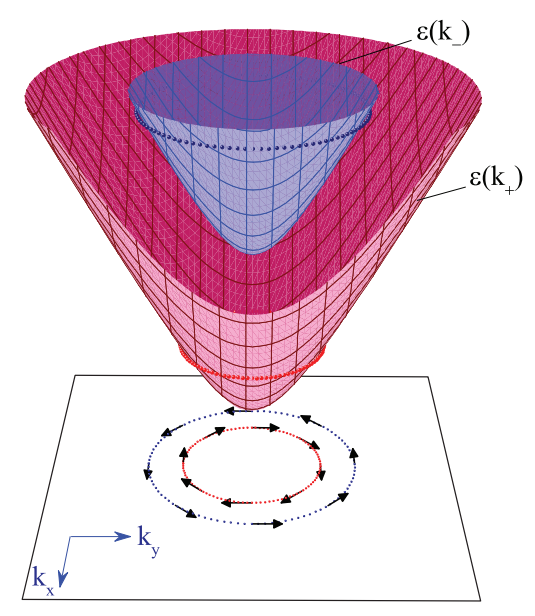

FIG. 2. (Color online) The energy surfaces of free electrons, in $k$ space, traveling in a pristine graphene sheet in the presence of spinorbit interactions. The contour lines trace out the two constant-energy surfaces that lie on different energy bands. The arrows show the electron spin direction. The spin of the electrons occupying different energy surfaces are opposite and is perpendicular to the electron momentum.

\section{SPIN-ORBIT COUPLINGS IN A GRAPHENE WIRE: $p-n-p$ STRUCTURE}

We now deal with the effect of SOCs on the bound states as well as on the transport properties of a graphene wire that can be created artificially by gating a flat graphene layer (see Fig. 3). The potential profile is in the form of a well, $V(x)=$ $V_{0}[\Theta(x)-\Theta(x-L)]$, with depth $V_{0}<0$ and width $L$. As previous we normalize the variables by using $x / L \rightarrow x$ which yields the potential $v(x)=v_{0}[\Theta(x)-\Theta(x-1)]$.

Derivation of the energy dispersion of bound states. An electron trapped within a graphene wire has spinor wave functions decaying exponentially outside the wire. Then, it is convenient to distinguish the wave vectors associated to the traveling and decaying wave functions which we denote by $k_{ \pm}$and $\alpha_{ \pm}$, respectively, and both are assumed to be tive real. We represent the spinor coefficients of the forward (backward) propagating wave functions by $A_{ \pm}^{f}\left(A_{ \pm}^{b}\right)$ where the spinors $A_{ \pm}^{f}$ are the same as in Eq. (13) but where $A_{ \pm}^{b}$ are now given by

$$
A_{ \pm}^{b}=\left(\begin{array}{c}
e^{-i \theta_{ \pm}} \\
i c_{ \pm} \\
\pm c_{ \pm} \\
\pm i e^{i \theta_{ \pm}}
\end{array}\right)
$$

The wave functions outside the wire, which satisfy the asymptotic boundary conditions $\psi(x \rightarrow \pm \infty)=0$ are as follows

$$
\psi(x<0)=B_{1} e^{\alpha_{+} x}\left(\begin{array}{c}
f_{+} \\
i c_{+}^{\prime} \\
c_{+}^{\prime} \\
i h_{+}
\end{array}\right)+B_{2} e^{\alpha_{-} x}\left(\begin{array}{c}
f_{-} \\
i c_{-}^{\prime} \\
-c_{-}^{\prime} \\
-i h_{-}
\end{array}\right),
$$

and

$$
\psi(1<x)=D_{1} e^{-\alpha_{+} x}\left(\begin{array}{c}
h_{+} \\
i c_{+}^{\prime} \\
c_{+}^{\prime} \\
i f_{+}
\end{array}\right)+D_{2} e^{-\alpha_{-} x}\left(\begin{array}{c}
h_{-} \\
i c_{-}^{\prime} \\
-c_{-}^{\prime} \\
-i f_{-}
\end{array}\right)
$$

where $f_{ \pm}=\left(k_{y}+\alpha_{ \pm}\right) /\left|k_{y}^{2}-\alpha_{ \pm}^{2}\right|^{1 / 2}, h_{ \pm}=\left(k_{y}-\alpha_{ \pm}\right) / \mid k_{y}^{2}-$ $\left.\alpha_{ \pm}^{2}\right|^{1 / 2}$ and $c_{ \pm}^{\prime}=(\varepsilon-\bar{\Delta}) /\left|k_{y}^{2}-\alpha_{ \pm}^{2}\right|^{1 / 2}$. In general, the electron wave inside the wire can be expressed as a linear combination of forward and backward traveling plane waves that consist of both, or at least one of, the $k_{ \pm}$states

$$
\begin{aligned}
\psi(0<x<1)= & C_{1} A_{+}^{f} e^{i k_{+} x}+C_{2} A_{+}^{b} e^{-i k_{+} x} \\
& +C_{3} A_{-}^{f} e^{i k_{-} x}+C_{4} A_{-}^{b} e^{-i k_{-} x} .
\end{aligned}
$$

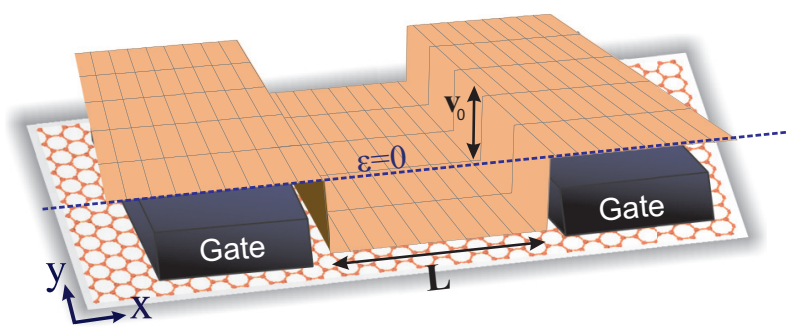

FIG. 3. (Color online) A schematic illustration of a graphene wire created by applying an external electrostatic potential. 
Since the electron is confined in the wire, the $x$ component of the probability current density should vanish, i.e., $j_{x}=$ $j_{x, \uparrow}+j_{x, \downarrow}=v_{F} \psi^{\dagger} \mathbf{S} \psi=0$ where $\mathbf{S}=\mathbf{1} \otimes \sigma_{x}$. This imposes a strong constraint on the wave function inside the well, resulting in the restriction that the solution is a standing wave.
Therefore, in the most general case, one finds the solution as $C_{1}=(a-i b) / 2, C_{2}=(a+i b) / 2, C_{3}\left(a^{\prime}-i b^{\prime}\right) / 2$ and $C_{4}=$ $\left(a^{\prime}+i b^{\prime}\right) / 2$, which ensures that the spinor components of the overall wave function in (18) are pure real or imaginary. This yields

$$
\psi(0<x<1)=\left(\begin{array}{c}
a \cos \left(k_{+} x+\theta_{+}\right)+b \sin \left(k_{+} x+\theta_{+}\right) \\
i c_{+}\left[a \cos \left(k_{+} x\right)+b \sin \left(k_{+} x\right)\right] \\
c_{+}\left[a \cos \left(k_{+} x\right)+b \sin \left(k_{+} x\right)\right] \\
i\left[a \cos \left(k_{+} x-\theta_{+}\right)+b \sin \left(k_{+} x-\theta_{+}\right)\right]
\end{array}\right)+\left(\begin{array}{c}
a^{\prime} \cos \left(k_{-} x+\theta_{-}\right)+b^{\prime} \sin \left(k_{-} x+\theta_{-}\right) \\
i c_{-}\left[a^{\prime} \cos \left(k_{-} x\right)+b^{\prime} \sin \left(k_{-} x\right)\right] \\
-c_{-}\left[a^{\prime} \cos \left(k_{-} x\right)+b^{\prime} \sin \left(k_{-} x\right)\right] \\
-i\left[a^{\prime} \cos \left(k_{-} x-\theta_{-}\right)+b^{\prime} \sin \left(k_{-} x-\theta_{-}\right)\right]
\end{array}\right) .
$$

Therefore, the first and third spinor components (related to sublattice index A) are pure real and the rest (corresponding to sublattice index B) are pure imaginary. Continuity of the wave function inside and outside the well gives the following transcendental equation for the exact dispersion equation

$$
\operatorname{det}\left|\begin{array}{cccccccc}
-f_{+} & -f_{-} & \cos \theta_{+} & \sin \theta_{+} & \cos \theta_{-} & \sin \theta_{-} & 0 & 0 \\
-c_{+}^{\prime} & -c_{-}^{\prime} & c_{+} & 0 & c_{-} & 0 & 0 & 0 \\
-c_{+}^{\prime} & c_{-}^{\prime} & c_{+} & 0 & -c_{-} & 0 & 0 & 0 \\
-h_{+} & h_{-} & \cos \theta_{+} & -\sin \theta_{+} & -\cos \theta_{-} & \sin \theta_{-} & 0 & 0 \\
0 & 0 & \cos \left(k_{+}+\theta_{+}\right) & \sin \left(k_{+}+\theta_{+}\right) & \cos \left(k_{-}+\theta_{-}\right) & \sin \left(k_{-}+\theta_{-}\right) & -h_{+} e^{-\alpha_{+}} & -h_{-} e^{-\alpha_{-}}=0 \\
0 & 0 & c_{+} \cos k_{+} & c_{+} \sin k_{+} & c_{-} \cos k_{-} & c_{-} \sin k_{-} & -c_{+}^{\prime} e^{-\alpha_{+}} & -c_{-}^{\prime} e^{-\alpha_{-}} \\
0 & 0 & c_{+} \cos k_{+} & c_{+} \sin k_{+} & -c_{-} \cos k_{-} & -c_{-} \sin k_{-} & -c_{+}^{\prime} e^{-\alpha_{+}} & c_{-}^{\prime} e^{-\alpha_{-}} \\
0 & 0 & \cos \left(k_{+}-\theta_{+}\right) & \sin \left(k_{+}-\theta_{+}\right) & -\cos \left(k_{-}-\theta_{-}\right) & -\sin \left(k_{-}-\theta_{-}\right) & -f_{+} e^{-\alpha_{+}} & f_{-} e^{-\alpha_{-}}
\end{array}\right|
$$

which we solved numerically.

Electron transmission. We turn to the study of the transmission across a graphene wire and calculate the fraction of the incident wave transmitted perpendicular to the wire. Assuming that the incident electron wave only consists of a $k_{+}$state we obtain the wave functions outside the wire as

$\psi(x<0)=A_{+}^{f} e^{i k_{+} x}+r_{++} A_{+}^{b} e^{-i k_{+} x}+r_{+-} A_{-}^{b} e^{-i k_{-} x}$,

and

$$
\psi(1<x)=t_{++} A_{+}^{f} e^{i k_{+} x}+t_{+-} A_{-}^{f} e^{i k_{-} x} .
$$

Here $r_{++}\left(r_{+-}\right)$is the amplitude of the reflected wave from the wire edge, and $t_{++}\left(t_{+-}\right)$denotes the amplitude of the transmitted wave with $k_{+}\left(k_{-}\right)$wave vector. The incoming and outgoing currents should be equal because the probability current is conserved. Using the electron density current $j_{x}=$ $v_{F} \psi^{\dagger} \mathbf{S} \psi$ together with the following relations

$$
\begin{aligned}
& \left(A_{ \pm}^{f}\right)^{\dagger} \mathbf{S} A_{\mp}^{f}=\left(A_{ \pm}^{b}\right)^{\dagger} \mathbf{S} A_{\mp}^{b}=\left(A_{ \pm}^{f}\right)^{\dagger} \mathbf{S} A_{ \pm}^{b}=0 \\
& \left(A_{ \pm}^{f}\right)^{\dagger} \mathbf{S} A_{ \pm}^{f}=-\left(A_{ \pm}^{b}\right)^{\dagger} \mathbf{S} A_{ \pm}^{b}=4 c_{ \pm} \sin \theta_{ \pm},
\end{aligned}
$$

we obtain the incoming and outgoing electron currents, in units of $v_{F}$, as follows

$$
\begin{aligned}
& \bar{j}_{x}(x<0)=4 c_{+} \sin \theta_{+}\left(1-r_{++}^{2}\right)-4 c_{-} \sin \theta_{-} r_{+-}^{2} \\
& \bar{j}_{x}(x>1)=4 c_{+} \sin \theta_{+} t_{++}^{2}-4 c_{-} \sin \theta_{-} t_{+-}^{2} .
\end{aligned}
$$

Note that $\theta_{ \pm}$is the angle that the incoming electron wave vector makes with the wire edge, i.e., $\theta_{ \pm} \in\left[\begin{array}{ll}0 & \pi\end{array}\right]$ because $k_{ \pm}$is positive. By imposing the continuity condition of the electron current we find

$$
c_{+} \sin \theta_{+}\left(1-r_{++}^{2}-t_{++}^{2}\right)-c_{-} \sin \theta_{-}\left(r_{+-}^{2}+t_{+-}^{2}\right)=0 .
$$

Continuing along the same lines for an incoming electron with the wave vector $k_{-}$we find

$$
c_{-} \sin \theta_{-}\left(1-r_{--}^{2}-t_{--}^{2}\right)-c_{+} \sin \theta_{+}\left(r_{-+}^{2}+t_{-+}^{2}\right)=0 .
$$

Combining Eqs. (25) and (26) for a mixed incoming current consisting of both $k_{+}$and $k_{-}$wave vectors yields the transmission and reflection probabilities as follows

$$
\begin{aligned}
& T=\frac{c_{+} \sin \theta_{+}\left(t_{++}^{2}+t_{-+}^{2}\right)+c_{-} \sin \theta_{-}\left(t_{--}^{2}+t_{-+}^{2}\right)}{c_{+} \sin \theta_{+}+c_{-} \sin \theta_{-}}, \\
& R=\frac{c_{+} \sin \theta_{+}\left(r_{++}^{2}+r_{-+}^{2}\right)+c_{-} \sin \theta_{-}\left(r_{--}^{2}+r_{-+}^{2}\right)}{c_{+} \sin \theta_{+}+c_{-} \sin \theta_{-}} .
\end{aligned}
$$

The coefficients of the reflected and transmitted electron waves can be easily obtained from the continuity condition of the wave function. If the wave function outside the wire decays for one of the available $k_{ \pm}$states, all the associated coefficients will be of course zero.

Extra Fabry-Pérot resonances. In Fig. 4 we summarize the results of our calculations performed for both the transmission spectrum and the bound states of a graphene wire with $V_{0}=$ $-60 \mathrm{meV}$ and $L=100 \mathrm{meV}$; i.e., $v_{0} \simeq-9.12$. The contour maps of the transmission spectrum show clear resonant peaks of Lorentzian form stretched to the oblique red boundaries separating the regions of the bound and unbound electron states. These resonant peaks show the regions at which the electron is completely transferred over the wire; that is exactly where the allowed energies of the bound states (with a standing 

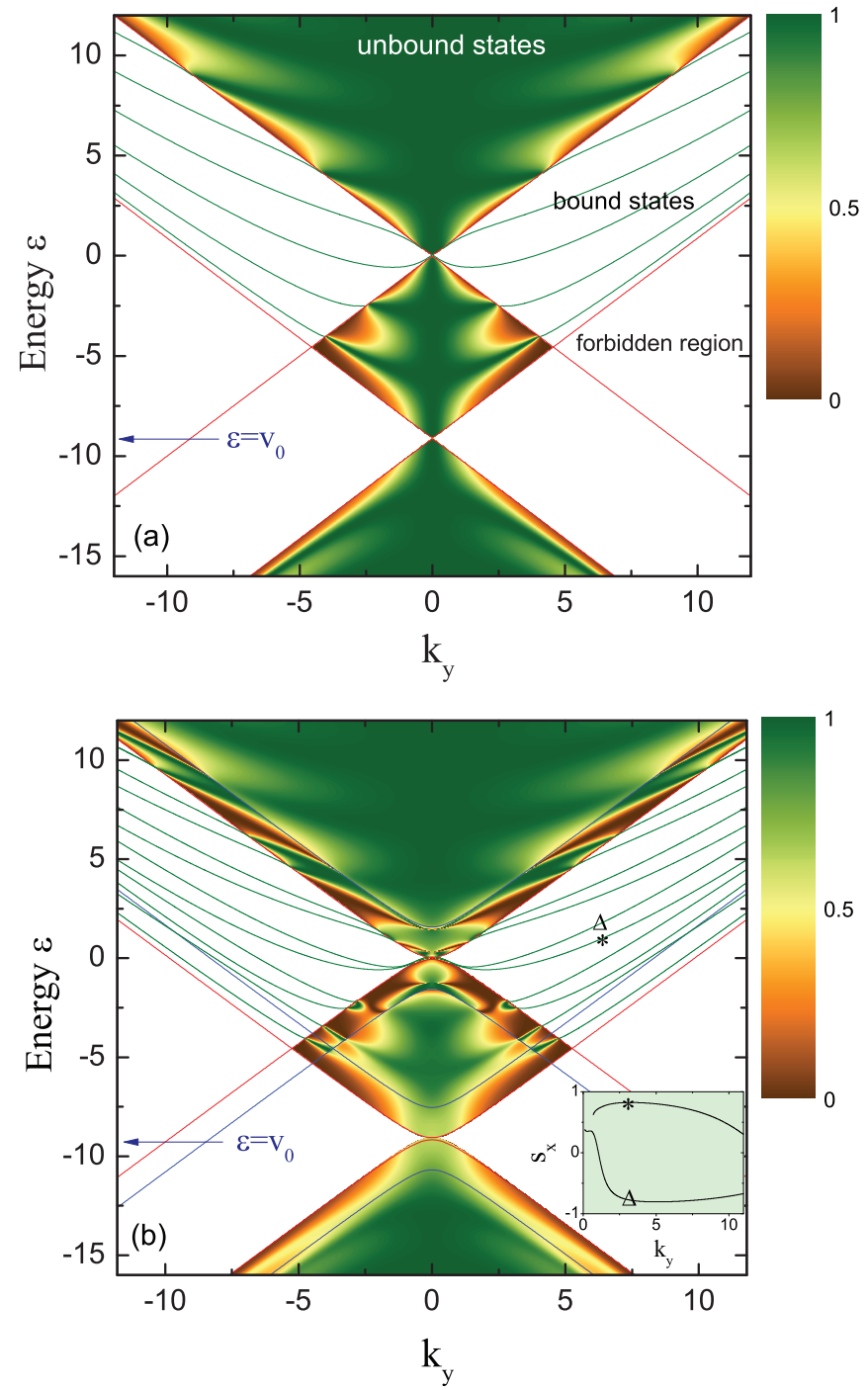

FIG. 4. (Color online) Contour plot of the transmission as a function of the energy and the $y$ component of the electron momentum for $v_{0}=-9.12, \bar{\Delta}=0.01$, and (a) $\bar{\lambda}=0$ and (b) $\bar{\lambda}=1.52$. The green solid curves are the allowed energies of the bound states. The red oblique lines separate the different regions related to the bound, unbound and forbidden electron states. In the region between the blue and red lines only one of the $k_{ \pm}$states is available for the electron transmission. In (a) the bound states are degenerate. The inset in (b) shows the average spin $S_{x}$ for two energy branches marked by the triangle and star symbols.

wave function inside the wire [see Eq. (18)]) cross the unbound states. $^{27,28}$ The same resonant peaks are observed in the transmission of light from the Fabry-Pérot optical resonators in which multiple reflections between two facing plates give rise to a standing wave inside the cavity resonator. In the presence of Rashba SOC the number of resonant peaks increases due to the spin-orbit splitting and, as a result, new channels open for electron transmission (for the case of pure intrinsic SOC no spin-orbit splitting occurs). Note that these extra resonant peaks appear in the region between the red and blue boundaries in Fig. 4(b). In this region there is only one $k_{+}$or $k_{-}$state providing a channel for electron transmission because the solution for the other $k$ state is an evanescent wave.
The $x$ component of the average spin is calculated for the two energy branches shown by the triangle and star marks [see the inset in Fig. 4(b)]. For an electron trapped inside the graphene wire the average spin only has a projection perpendicular to the graphene wire and the other normal components are zero. In this special case the electron travels along the graphene wire and its spin is perpendicular to the electron motion.

\section{SPIN-ORBIT INTERACTIONS IN A SUPERLATTICE: PERIODIC $p$ - $n$ STRUCTURE}

We consider an ideal array of periodically spaced electrical gates, forming a regular superlattice of graphene wires arranged along the $x$ direction (see Fig. 5). We approximate the superlattice potential by an infinite series of rectangular barriers as follows

$V(x)=\sum_{n=-\infty}^{\infty} V_{0}[\Theta(x-w+n L)-\Theta(x+(n-1) L)]-\frac{V_{0}}{2}$,

where $L=w+b$ is the superlattice period, and $w$ and $b$ denote the width of the well and the barrier, respectively. We use dimensionless parameters as before and replace $w / L \rightarrow w$ and $b / L \rightarrow b$. The translation operator which shifts the wave function argument by a superlattice period can be expressed as

$$
T=T_{w} T_{b}
$$

where $T_{w}=\left.M G_{w} M^{-1}\right|_{v=-\frac{v_{0}}{2}}$ and $T_{b}=\left.M G_{b} M^{-1}\right|_{v=\frac{v_{0}}{2}}$ transfer the wave function between the two edges of a well and a barrier, respectively. The operators $M, G_{w}$, and $G_{b}$, which functionally depend on $v$, are given by

$$
M[v]=\left(\begin{array}{cccc}
e^{i \theta_{+}} & e^{i \theta_{-}} & e^{-i \theta_{+}} & e^{-i \theta_{-}} \\
i c_{+} & i c_{-} & i c_{+} & i c_{-} \\
c_{+} & -c_{-} & c_{+} & -c_{-} \\
i e^{-i \theta_{+}} & -i e^{-i \theta_{-}} & i e^{i \theta_{+}} & -i e^{i \theta_{-}}
\end{array}\right),
$$

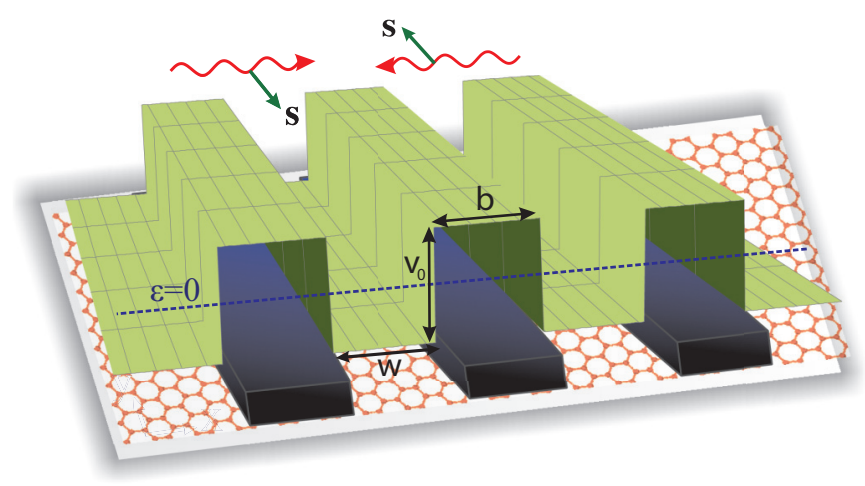

FIG. 5. (Color online) A schematic illustration of a superlattice of graphene wires created by an ideal array of periodically spaced electrical gates. The electron spin is normal to its momentum if the electron travels perpendicular to the superlattice as illustrated in the figure. 
and

$$
G_{w(b)}[v]=\left(\begin{array}{cccc}
e^{i k_{+} w(b)} & 0 & 0 & 0 \\
0 & e^{i k_{-} w(b)} & 0 & 0 \\
0 & 0 & e^{-i k_{+} w(b)} & 0 \\
0 & 0 & 0 & e^{-i k_{-} w(b)}
\end{array}\right) .
$$

Note that all the parameters $\theta_{ \pm}, c_{ \pm}$, and $k_{ \pm}$depend on both $\varepsilon$ and $v$. The wave function transferred by $T$ should satisfy the Bloch condition $\psi(x+L)=e^{i k_{x} L} \psi(x)$. For a given Bloch momentum $k_{x}$ this imposes a rigorous restriction on the $\varepsilon$ dependent eigenvalues of $T$ because the act of $T$ on the wave function should give the Bloch phase $e^{ \pm i k_{x} L}$. Therefore one obtains an exact energy dispersion equation as the real part of the eigenvalues should be equal to $\cos \left(k_{x} L\right)$.

Energy spectrum and spin configuration. In Fig. 6(a) we show the lowest electron-hole energy surfaces, in $k$ space, obtained by including the effect of spin-orbit interactions. In Ref. 29 it was shown that in a superlattice on graphene extra Dirac cones appear in the energy spectrum in addition to the main Dirac cone. The Rashba spin-orbit interaction splits the energy surfaces in the direction of the array of barriers, resulting in additional Dirac cones in the energy spectrum. Notice that the Rashba SOC strongly deforms the linear dispersion of the main Dirac cone in the $x$ direction but less in the $y$ direction. The intrinsic spin-orbit interaction is responsible for the energy gaps in the electron-hole energy surfaces around the Dirac points. In contrast to the case of a graphene layer without periodic potential, the inclusion of Rashba SOC does not remove these energy gaps.

Figure 6(b) displays the spin configuration in $k$ space calculated for the lowest electron energy surface. In contrast to the case without superlattice potential we find that the electron spin is no longer perpendicular to its momentum. If the electrons travel strictly along the array of barriers $\left(k_{y}=0\right)$ the Hamiltonian commutes with the unitary operator

$$
u=\left(\begin{array}{cccc}
0 & 0 & 0 & -i \\
0 & 0 & -i & 0 \\
0 & i & 0 & 0 \\
i & 0 & 0 & 0
\end{array}\right)
$$

Since the eigenvalues of $u$ are \pm 1 , consequently, one finds from the relations $u S_{y} u=S_{y}, u S_{x} u=-S_{x}$ and $u S_{z} u=-S_{z}$ that only the $y$ component of the average spin, $S_{y}$, is nonzero. In order to extract the symmetries of the spin configuration in a more efficient way, we present in Fig. 7 a contour plot of the average spin components calculated for the electrons occupying the lowest conduction band around the Dirac points. From the results we find that by a mirror reflection of the direction of motion with respect to the $k$-space axes the spin component perpendicular to the reflection axis is inverted: $S_{x}\left(k_{x} \hat{e}_{x}+k_{y} \hat{e}_{y}\right)=-S_{x}\left(k_{x} \hat{e}_{x}-k_{y} \hat{e}_{y}\right)$ and $S_{y}\left(k_{x} \hat{e}_{x}+k_{y} \hat{e}_{y}\right)=$ $-S_{y}\left(-k_{x} \hat{e}_{x}+k_{y} \hat{e}_{y}\right)$. Thus, the electrons traveling in opposite directions possess antiparallel spins; that is $\hat{S}(\vec{k})=-\hat{S}(-\vec{k})$. Therefore, the average spin of a completely filled energy surface is zero.

Density of energy states. When the graphene superlattice involves spin-orbit couplings, the number of $k$ states available

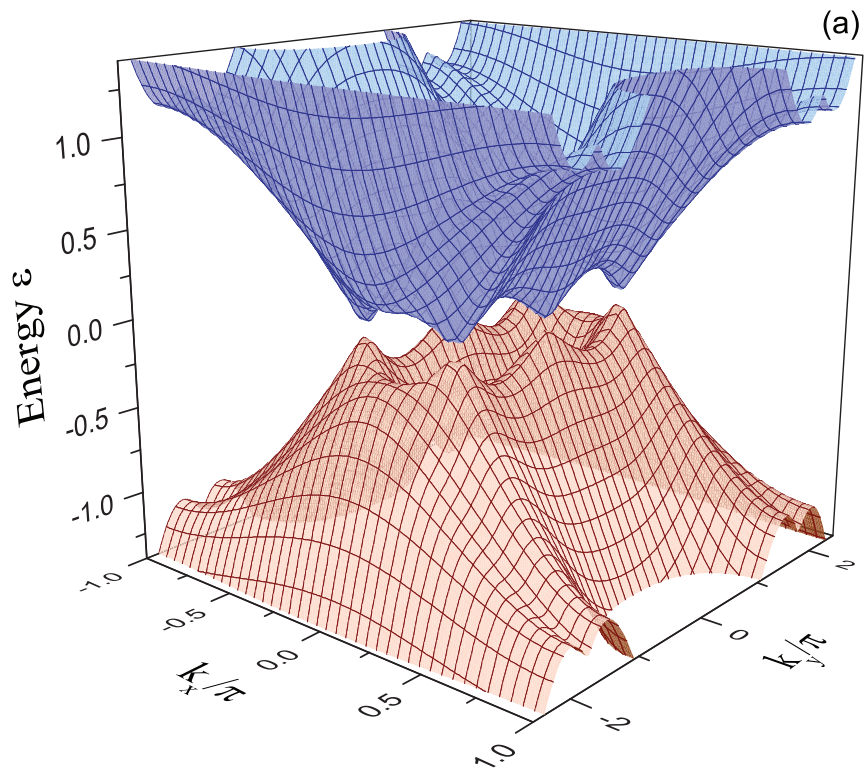

(a)

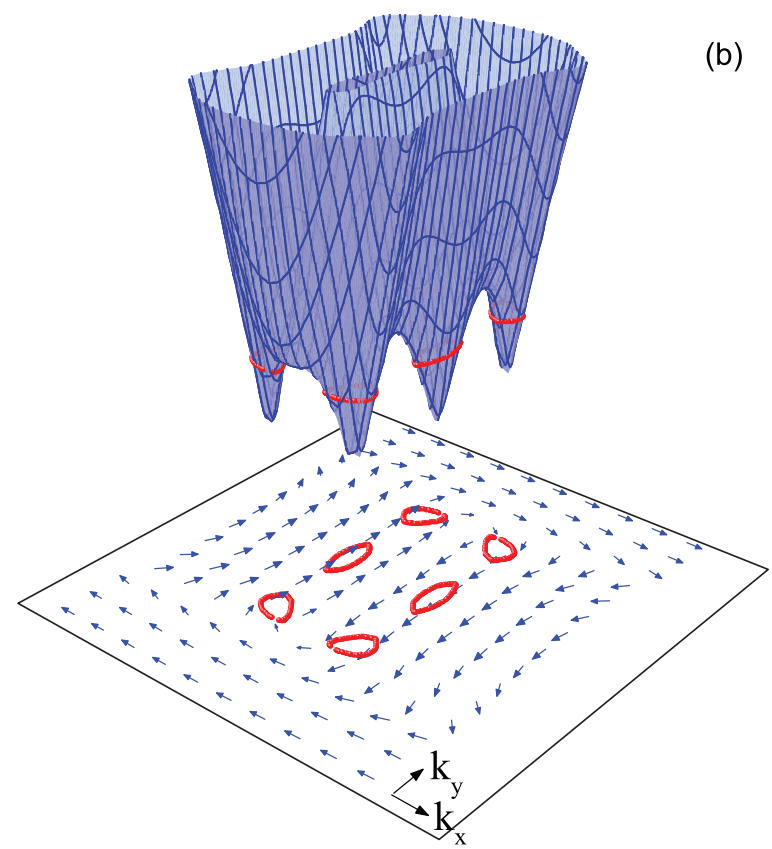

FIG. 6. (Color online) (a) The lowest electron-hole energy surfaces of the graphene superlattice in the presence of spin-orbit interactions. The strengths of SOCs are $\bar{\Delta}=0.01$ and $\bar{\lambda}=1.52$, the normalized depth and width of the potential wells are taken as $v_{0}=$ 15.2 and $w=0.5$, respectively, corresponding to $V_{0}=100 \mathrm{meV}$, $w=50 \mathrm{~nm}$ and the superlattice period $L=100 \mathrm{~nm}$. (b) Projection of the spin configuration for electrons in the lowest conduction band. The red curves correspond to the constant energy surface $\varepsilon=0.16$.

for the occupancy of electrons per unit volume and energy is given by

$$
D(E)=\sum_{n, s, \vec{k}} \delta\left[E-E_{n, s}(\vec{k})\right] .
$$

To calculate numerically the density of states (DOS) we replace the $\delta$ function by a sharp Gaussian broadening 

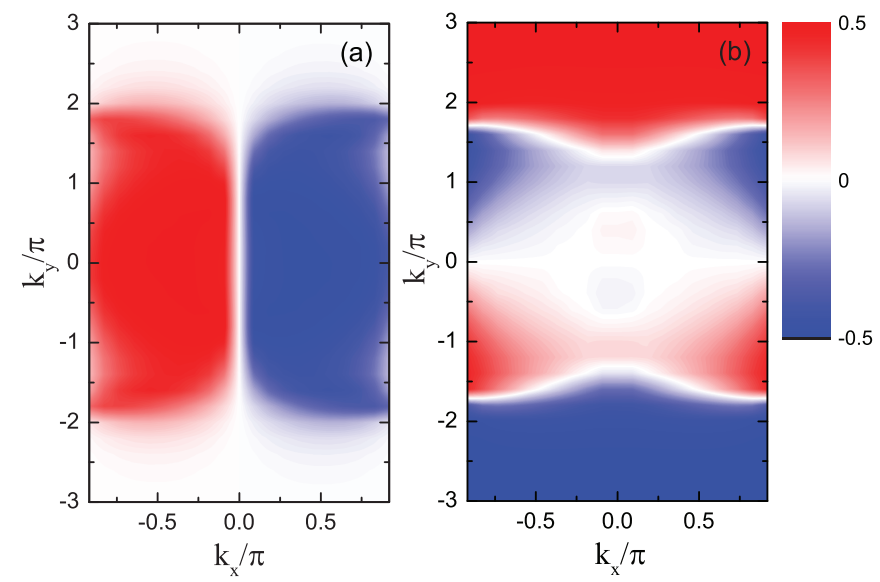

FIG. 7. (Color online) Contour plot of the average spin components $S_{y}$ (a) and $S_{x}$ (b) calculated for the lowest conduction band of a graphene superlattice around the Dirac cones.

$\delta\left[E-E_{n, s}(\vec{k})\right] \rightarrow \frac{1}{\Gamma \sqrt{\pi}} \exp \left\{-\frac{1}{\Gamma^{2}}\left[E-E_{n, s}(\vec{k})\right]\right\}$, where $\Gamma$ determines the amount of the broadening. In Fig. 8, the DOS of the graphene superlattice is calculated for $V_{0}=100 \mathrm{meV}$ and $L=100 \mathrm{~nm}$ by taking spin-orbit couplings into account (dashed curves) and the results are compared with the case of a graphene superlattice in the absence of spin-orbit interactions (solid curves). The dotted black curve shows the DOS of a flat pristine graphene sheet with SOCs strengths $\bar{\Delta}=0.01$ and $\bar{\lambda}=1.52$. With the inclusion of Rashba spin-orbit interaction the DOS exhibits an oscillating structure between the peaks and dips of the curves obtained in the absence of spin-orbit couplings. The fluctuations originate from the new saddle points in the energy spectrum due to the Rashba spin-orbit splitting around the Dirac points. The major oscillations at low energies arise due to the supperlattice potential, in particular

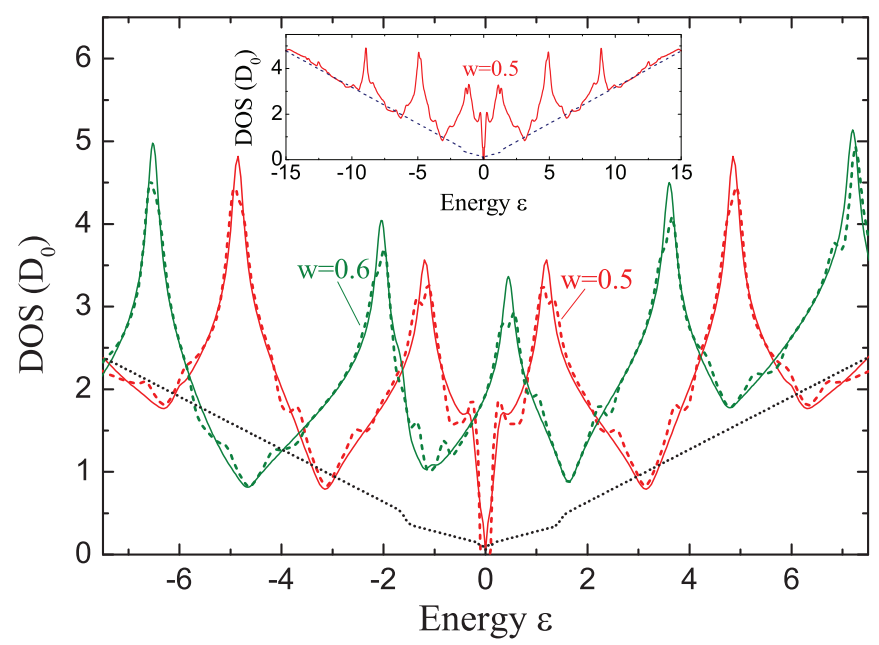

FIG. 8. (Color online) The density of energy states of a graphene superlattice with $v_{0}=15.2$ in the absence (solid curves) and presence (dashed curves) of spin-orbit couplings. The results are shown, in units of $D_{0}=L / \hbar v_{F}$, for $w=0.5$ and $w=0.6$ by the red and green curves, respectively. The DOS without the superlattice potential is represented by the black dotted curve for $\bar{\Delta}=0.01$ and $\bar{\lambda}=1.52$. The inset shows the DOS in a wider energy range (up to the barrier height) for $w=0.5$. below the barrier height, but with increasing energy the influence of the superlattice potential diminishes, resulting in a quenching of the oscillations arising from the Rashba SOC. For $w=0.6$ the electron-hole symmetry is broken ${ }^{29}$ and the touching point of the valence and conduction bands moves away from zero energy and hence the DOS has no zero at $\varepsilon=0$ (see the green curves in Fig. 8). Moreover, the results show that changing the width of the potential wells has no qualitative consequences on the feature of oscillating structure induced by SOCs.

Low-temperature diffusive conductivity. At sufficiently low temperatures the inelastic scattering due to thermal vibrations of the atoms has negligible contribution to the electron transport and the tensor elements of the diffusive DC conductivity is given by a sum of the contributions from each energy band:

$$
\sigma_{\mu \nu}=\frac{e^{2} v_{F} \beta}{A} \sum_{n, s, \vec{k}} \tau v_{n, s}^{\mu} v_{n, s}^{v} f\left[E_{n, s}(\vec{k})\right]\left\{1-f\left[E_{n, s}(\vec{k})\right]\right\} .
$$

Here $\beta=1 / k_{B} T, \quad A$ is the area of the system, $\vec{v}_{n, s}=\frac{1}{\hbar} \partial E_{n, s} / \partial \vec{k}$ is the electron velocity, $f\left(E_{n, s}(\vec{k})\right)=$ $1 /\left\{\exp \left[\beta\left(E_{F}-E_{n, s}(\vec{k})\right)\right]+1\right\}$ is the equilibrium Fermi-Dirac distribution function, and $\tau$ denotes the momentum relaxation time which, at low temperatures, we assume to be a constant near the Fermi energy $\left(\tau \approx \tau_{F}\right)$. Note that the isotropy of the diffusive conductivity is not influenced by the spinorbit interactions as the conductivity tensor contains only the diagonal components $\sigma_{x x}$ and $\sigma_{y y}$. This is due to the symmetry of the energy surfaces that results in the inversion symmetry of the electron velocity as $v_{n, s}^{x}(-\vec{k}) v_{n, s}^{y}(-\vec{k})=$ $-v_{n,-s}^{x}(\vec{k}) v_{n,-s}^{y}(\vec{k})$. Since the sum in Eq. (35) runs over all $k$ states the off-diagonal components of the conductivity vanish. Meanwhile, the isotropy of the diffusive conductivity for a pristine graphene sheet including spin-orbit couplings is also preserved.

To illustrate the effect of spin-orbit interactions on the electrical conductivity of a graphene superlattice we plot the tensor components $\sigma_{x x}$ and $\sigma_{y y}$ by assuming that temperature is low enough $(T=4 \mathrm{~K})$. The results are shown in Fig. 9 for $w=0.5$ and $w=0.6$. Comparing the conductivities $\sigma_{x x}$ and $\sigma_{y y}$ we find that the Rashba spin-orbit splitting has a more pronounced effect on the conductivity component parallel to the barriers. The spin-splitting due to the Rashba SOC alters the electron velocity especially around the Dirac points. These Dirac points were located at $k_{x}=0$ before the spin-splitting, ${ }^{29}$ but now each point is moved from $k_{x}=0$ to the two side points and as a result the velocity component $v_{n, s}^{x}(\vec{k})$ is more affected by the SOC than the normal component $v_{n, s}^{y}(\vec{k})$. Therefore, additional local oscillations appear in the conductivity $\sigma_{x x}$ in addition to the main oscillations resulting from the superlattice potential. Moreover, for both widths of the potential wells the Rashba SOC decreases the conductivity tensor component $\sigma_{x x}$.

\section{CONCLUSIONS}

Within the continuum Dirac equation we studied the effect of SOCs in a graphene sheet with different potential structures: (i) a pristine graphene sheet, (ii) a graphene wire ( $p-n-p$ 

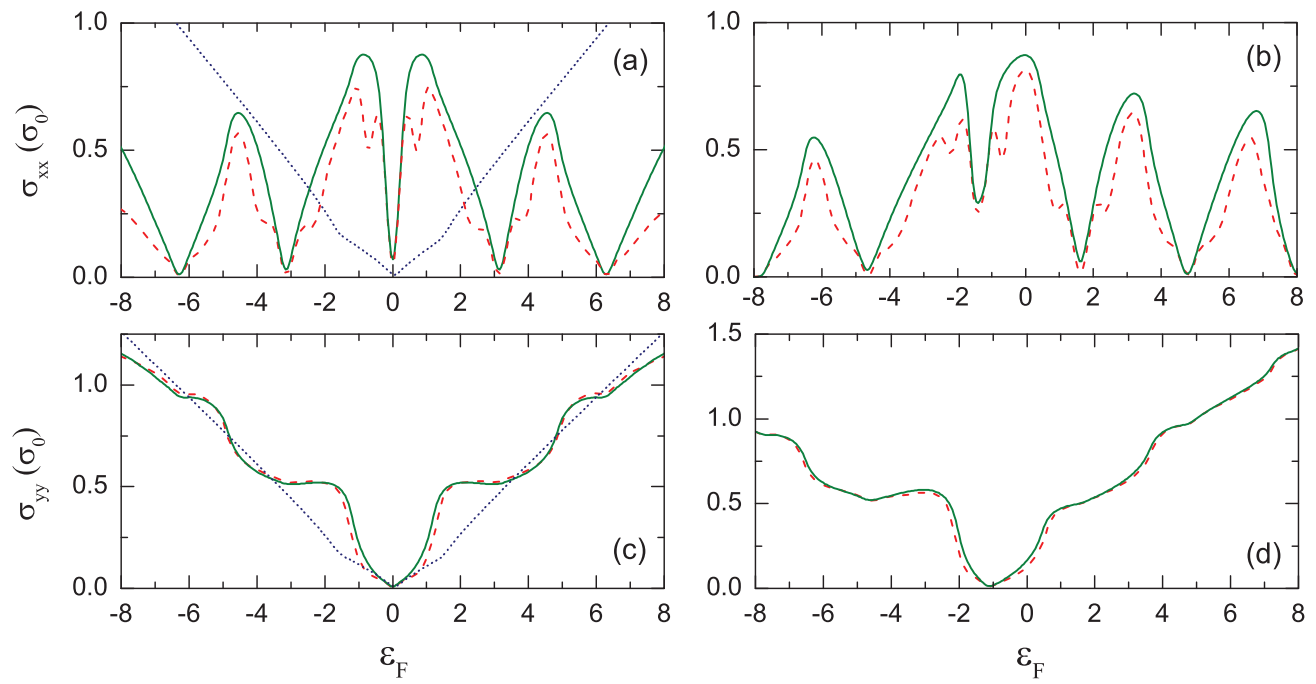

FIG. 9. (Color online) (a) and (b) show the diffusive conductivity $\sigma_{x x}$, in units of $\sigma_{0}=e^{2} v_{F} \tau_{F} / \hbar L$, for a graphene superlattice with $w=0.5$ and $w=0.6$, respectively. (c) and (d) are the same as in (a), (b) but now for the conductivity $\sigma_{y y}$. The solid (dashed) curves indicate the conductivities in the absence (presence) of SOCs. The blue dotted curve in (a) and (c) correspond to the conductivity of a graphene sheet without superlattice potential.

structure), and (iii) a graphene superlattice. In the latter two structures an external electrostatic potential is applied to the graphene sheet inducing artificially quantum confinement for Dirac fermions. The energy dispersion relation of a Dirac fermion confined in each structure is analytically derived. We found that in a graphene sheet without any potential the interplay between the Rashba and intrinsic SOCs breaks the symmetry of the electron-hole energy dispersion as long as the strength of one SOC mechanism becomes zero. The Rashba SOC results in spin-orbit splitting of the energy spectrum with the two spin configurations for the electron being perpendicular to its motion. However, this is no longer the case when an external electrostatic potential is applied; i.e., spin can have a component parallel to the electron motion. Our results demonstrate that the Rashba spin-orbit splitting doubles the number of Fabry-Pérot resonant peaks in the transmission spectrum of a graphene wire and, as a result, new channels open that are able to transfer electrons. Moreover, it is shown that due to the presence of Rashba SOC extra spin split Dirac cones appear in the energy spectrum of a graphene superlattice, leading to the appearance of small local fluctuations in the density of energy states as well as in the diffusive conductivity.

\section{ACKNOWLEDGMENTS}

This work was supported by the Flemish Science Foundation (FWO-Vl) and the European Science Foundation (ESF) under the EUROCORES Program EuroGRAPHENE within the project CONGRAN. The generous support provided by the Saudi Center for Theoretical Physics (SCTP) is highly appreciated by A.J. and E.B.C. They also thank the Deanship of Scientific Research at King Faisal University for funding this work under the Project No. 130193. *kh.shakoory@gmail.com

†a.jellal@ucd.ac.ma

${ }^{1}$ H. Dery, H. Wu, B. Ciftcioglu, M. Huang, Y. Song, R. Kawakami, J. Shi, I. Krivorotov, I. Žutić, and L. J. Sham, IEEE Trans. Electron Devices 59, 259 (2012).

${ }^{2}$ K. S. Novoselov, A. K. Geim, S. V. Morozov, D. Jiang, M. I. Katsnelson, I. V. Grigorieva, S. V. Dubonos, and A. A. Firsov, Nature (London) 438, 197 (2005).

${ }^{3}$ Y. Zheng, Y. W. Tan, H. L. Stormer, and P. Kim, Nature (London) 438, 201 (2005).

${ }^{4}$ N. Tombros, C. Jozsa, M. Popinciuc, H. T. Jonkman, and B. J. van Wees, Nature (London) 448, 571 (2007).

${ }^{5}$ C. Ertler, S. Konschuh, M. Gmitra, and J. Fabian, Phys. Rev. B 80, 041405 (2009).

${ }^{6}$ D. Huertas-Hernando, F. Guinea, and A. Brataas, Phys. Rev. Lett. 103, 146801 (2009).
${ }^{7}$ M. Ohishi, M. Shiraishi, R. Nouchi, T. Nozaki, T. Shinjo, and Y. Suzuki, Jpn. J. Appl. Phys. 46, L605 (2007).

${ }^{8}$ C. Józsa, M. Popinciuc, N. Tombros, H. T. Jonkman, and B. J. van Wees, Phys. Rev. B 79, 081402 (2009).

${ }^{9}$ M. Popinciuc, C. Józsa, P. J. Zomer, N. Tombros, A. Veligura, H. T. Jonkman, and B. J. van Wees, Phys. Rev. B 80, 214427 (2009).

${ }^{10}$ W. Han, K. Pi, W. H. Wang, K. M. McCreary, Y. Li, W. Bao, P. Wei, J. Shi, C. N. Lau, and R. K. Kawakami, Proc. SPIE 7398, 739819 (2009).

${ }^{11}$ M. Nishioka and A. M. Goldman, Appl. Phys. Lett. 90, 252505 (2007).

${ }^{12}$ T. Maassen, F. K. Dejene, M. H. D. Guimarães, C. Józsa, and B. J. van Wees, Phys. Rev. B 83, 115410 (2011).

${ }^{13}$ C. L. Kane and E. J. Mele, Phys. Rev. Lett. 95, 226801 (2005).

${ }^{14}$ Z. Qiao, W.-K. Tse, H. Jiang, Y. Yao, and Q. Niu, Phys. Rev. Lett. 107, 256801 (2011). 
${ }^{15}$ F. Mireles and J. Schliemann, New J. Phys. 14, 093026 (2012).

${ }^{16}$ H. Zhang, C. Lazo, S. Blügel, S. Heinze, and Y. Mokrousov, Phys. Rev. Lett. 108, 056802 (2012).

${ }^{17}$ Y. Yao, F. Ye, X.-L. Qi, S.-C. Zhang, and Z. Fang, Phys. Rev. B 75, 041401 (2007).

${ }^{18}$ M. Gmitra, S. Konschuh, C. Ertler, C. Ambrosch-Draxl, and J. Fabian, Phys. Rev. B 80, 235431 (2009).

${ }^{19}$ S. Konschuh, M. Gmitra, and J. Fabian, Phys. Rev. B 82, 245412 (2010).

${ }^{20}$ J. C. Boettger and S. B. Trickey, Phys. Rev. B 75, 121402(R) (2007).

${ }^{21}$ Y. S. Dedkov, M. Fonin, U. Rüdiger, and C. Laubschat, Phys. Rev. Lett. 100, 107602 (2008).

${ }^{22}$ O. Rader, A. Varykhalov, J. Sánchez-Barriga, D. Marchenko, A. Rybkin, and A. M. Shikin, Phys. Rev. Lett. 102, 057602 (2009).
${ }^{23}$ Z. Y. Li, Z. Q. Yang, S. Qiao, J. Hu, and R. Q. Wu, J. Phys.: Condens. Matter 23, 225502 (2011).

${ }^{24}$ D. Marchenko, A. Varykhalov, M. R. Scholz, G. Bihlmayer, E. I. Rashba, A. Rybkin, A. M. Shikin, and O. Rader, Nature Commun. 3, 1232 (2012).

${ }^{25}$ A. H. Castro Neto and F. Guinea, Phys. Rev. Lett. 103, 026804 (2009).

${ }^{26}$ S. Abdelouahed, A. Ernst, J. Henk, I. V. Maznichenko, and I. Mertig, Phys. Rev. B 82, 125424 (2010).

${ }^{27}$ M. Ramezani Masir, P. Vasilopoulos, and F. M. Peeters, Phys. Rev. B 82, 115417 (2010).

${ }^{28}$ J. M. Pereira, Jr., V. Mlinar, F. M. Peeters, and P. Vasilopoulos, Phys. Rev. B 74, 045424 (2006).

${ }^{29}$ M. Barbier, P. Vasilopoulos, and F. M. Peeters, Phys. Rev. B 81, 075438 (2010). 